\title{
Impact of cation-based localized electronic states on the conduction and valence band structure of $\mathrm{Al}_{1-x} \mathrm{In}_{x} \mathrm{~N}$ alloys
}

\author{
S. Schulz, , 因 M. A. Caro, ${ }^{1,2}$ and E. P. O'Reilly ${ }^{1,2}$ \\ ${ }^{1}$ Photonics Theory Group, Tyndall National Institute, Dyke Parade, Cork, Ireland \\ ${ }^{2}$ Department of Physics, University College Cork, Cork, Ireland
}

(Dated: June 16, 2021)

\begin{abstract}
We demonstrate that cation-related localized states strongly perturb the band structure of $\mathrm{Al}_{1-x} \operatorname{In}_{x} \mathrm{~N}$ leading to a strong band gap bowing at low In content. Our first-principles calculations show that In-related localized states are formed both in the conduction and the valence band in $\mathrm{Al}_{1-x} \operatorname{In}_{x} \mathrm{~N}$ for low In composition, $x$, and that these localized states dominate the evolution of the band structure with increasing $x$. Therefore, the commonly used assumption of a single composition-independent bowing parameter breaks down when describing the evolution both of the conduction and of the valence band edge in $\mathrm{Al}_{1-x} \operatorname{In}_{x} \mathrm{~N}$.

PACS numbers: 74.70.Dd, 78.55.Cr, 71.20.Nr
\end{abstract}

The semiconductor alloy $\mathrm{Al}_{1-x} \mathrm{In}_{x} \mathrm{~N}$ has attracted extensive research interest due to its potential to improve the performance of electronic and optoelectronic devices. For high-frequency and high-power microwave applications, high-electron-mobility transistors based on $\mathrm{Al}_{1-x} \operatorname{In}_{x} \mathrm{~N} / \mathrm{GaN}$ have been considered recently $\underline{\underline{\underline{1}}}$ Since the band gaps of AlN and $\mathrm{InN}$ are $6.25 \mathrm{eV}$ and 0.69 $\mathrm{eV}$, respectively $\underset{2}{2}$ low $\mathrm{InN}$ compositions are required to achieve deep UV emission. However, basic and central properties of $\mathrm{Al}_{1-x} \mathrm{In}_{x} \mathrm{~N}$ alloys are not well known or understood. For example, when designing optoelectronic devices based on $\mathrm{Al}_{1-x} \operatorname{In}_{x} \mathrm{~N}$ alloys, knowledge about the energy gap variation of $\mathrm{Al}_{1-x} \operatorname{In}_{x} \mathrm{~N}$ with composition $x$ is of central importance. It is common for most group III-V materials to assume that the band gap of an alloy $A_{1-x} B_{x} C$ has a close to linear variation with composition $x$, with any deviation from linearity described by a small quadratic term, $-b x(1-x)$, where $b$ is the bowing parameter $\stackrel{3}{ }$ :

$$
E_{g}^{A B C}(x)=(1-x) E_{g}^{A C}(x)+x E_{g}^{B C}(x)-b x(1-x) .
$$

Here $A$ and $B$ denote cations while $C$ denotes the anion. For AlInN this simple assumption has been questioned by several groups, based on experimental data $\stackrel{\underline{4}}{\underline{1}}$ To date, the physical origin of this behavior is unclear.

In this letter we propose and demonstrate that cationrelated localized states strongly perturb the band structure of the III-V alloy $\mathrm{Al}_{1-x} \operatorname{In}_{x} \mathrm{~N}$. We show that Inrelated localized states are formed both in the conduction band $(\mathrm{CB})$ and the valence band $(\mathrm{VB})$ in $\mathrm{Al}_{1-x} \mathrm{In}_{x} \mathrm{~N}$ for low In composition $x$, and that these localized states dominate the evolution of the band structure with increasing $x$. This is initially surprising - to date the band structure of all III-V materials involving alloying between elements from the third to the fifth row of the periodic table has been described using a single compositionindependent bowing parameter $b . \frac{3}{=}$ In addition, the band gap bowing in the two ternary alloys GaInN and AlGaN is generally well described using Eq. (10), but with GaInN showing some deviations from this simple description $\underline{6.7}^{6.7}$
A similar behavior might therefore also be expected for $\mathrm{Al}_{1-x} \operatorname{In}_{x} \mathrm{~N}$. We note however that $\mathrm{AlInN}$ has been reported to have a very large and composition-dependent bowing parameter, with values of $b$ ranging from $\sim 2.5 \mathrm{eV}$ (high In content) $\underline{8}$ to $10.3 \mathrm{eV}$ (low In content) $\underline{\underline{9}}$ A large bowing parameter has to date generally been associated with the presence of isoelectronic states in a semiconductor alloy, such as $\mathrm{ZnTe}_{1-x} \mathrm{Se}_{x} \stackrel{10}{\underline{10}}$ Using density functional theory (DFT), we show here that this is also the case for $\mathrm{Al}_{1-x} \operatorname{In}_{x} \mathrm{~N}$, and that the evolution of the band structure of $\mathrm{Al}_{1-x} \operatorname{In}_{x} \mathrm{~N}$ for values of $x$ as large as $\sim 20 \%$ is dominated by In-related localized isoelectronic defect levels. This behavior arises due to the marked difference between the band structure of AlN and $\mathrm{InN}$, including a difference in energy gap of over $5 \mathrm{eV}$. Most of this difference in energy gap between free-standing $\mathrm{AlN}$ and $\mathrm{InN}$ occurs in the $\mathrm{CB}$, with the estimated $\mathrm{CB}$ offset varying from $4.0 \mathrm{eV}^{11}$ to $4.6 \mathrm{eV}^{7}$. This compares with estimated $\mathrm{CB}$ offsets of 1.4-2.7 eV between AlN and GaN,, $12-14$ and of 1.6-2.3 eV between $\mathrm{GaN}$ and $\mathrm{InN}, \underline{7,13,15}$

We show here by explicit calculation that the large difference in the $\mathrm{CB}$ energies of $\mathrm{InN}$ and $\mathrm{AlN}$ leads to the formation of a localized resonant defect state above the $\mathrm{CB}$ edge (CBE) when a single $\mathrm{Al}$ atom is replaced by an In atom in AlN. We further show that the interaction between In-related localized states and the AlN host matrix states then leads to a rapid reduction in the CBE energy of $\mathrm{Al}_{1-x} \operatorname{In}_{x} \mathrm{~N}$ with increasing In composition $x$, analogous to the behavior of alloys such as $\mathrm{ZnTe}_{1-x} \mathrm{Se}_{x}, 10$

Turning to the VB states, we note that several studies have shown how the highest valence states tend to become localized in GaInN alloys $\underline{\underline{16}-20}$ We show that the highest VB states can be strongly localized in AlInN, undertaking DFT studies both on ordered and on disordered supercells (SCs). We first consider ordered SCs, in which each In atom only has $\mathrm{Al}$ atoms as second nearest neighbors. We show that the VB edge (VBE) energy has a much weaker variation with composition in such structures compared with that which is observed in structures containing In pairs and clusters, where an In 


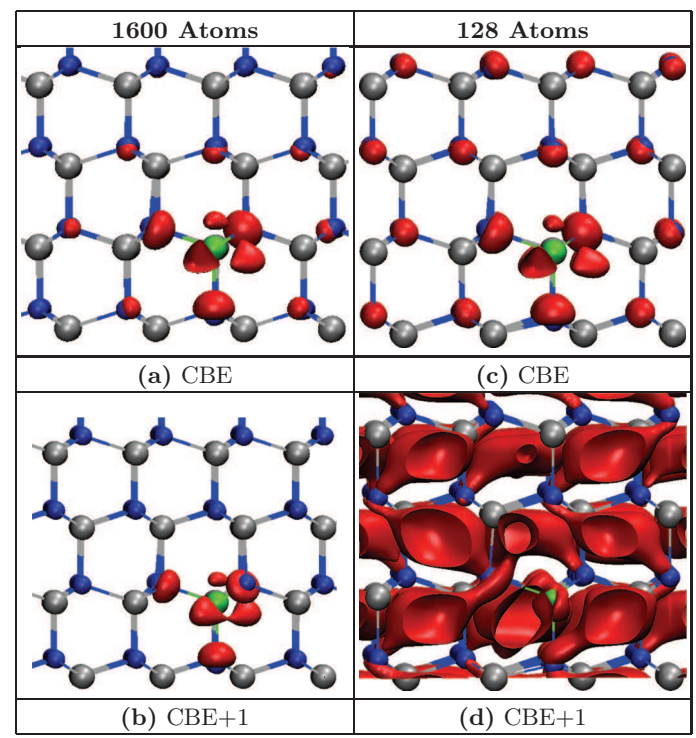

FIG. 1. (Color online) Charge densities for $\mathrm{CBE}$ and $\mathrm{CBE}+1$ at $\mathbf{k}=\mathbf{0}$ obtained within LDA- (left) and HSE-DFT (right) calculations. The dark (red) isosurface of the charge density corresponds to $10 \%$ of the maximum value for the given eigenstate. SCs contain 1600 (LDA) and 128 (HSE) atoms, respectively.

pair is formed when a $\mathrm{N}$ atom has two In neighbors. We find that even a single In pair in a SC can strongly shift the VBE energy upwards. It also tends to localize the highest valence state, consistent with In pairs introducing localized states below the VBE in $\mathrm{Al}_{1-x} \operatorname{In}_{x} \mathrm{~N}$.

To analyze the impact of a single, isolated In atom in an AlN matrix, we first present the results of DFT calculations undertaken using the local density approximation (LDA) and the plane-wave-based ab initio package VASP $\stackrel{21}{=}$ SCs with 1600 atoms have been studied. The energy cutoff for the plane waves was $400 \mathrm{eV}$, the semi-core $d$ electrons of In have been treated as valence electrons and $\mathbf{k}=\mathbf{0}$ has been chosen. The structure is relaxed using a valence force field (VFF) model $\underline{6,22}$ Figure 1 shows the calculated charge densities of (a) the $\mathrm{CBE}$ and (b) the first excited $\mathrm{CB}$ state $(\mathrm{CBE}+1)$ at $\mathbf{k}=\mathbf{0}$. Figure $1(\mathrm{~b})$ shows explicitly that a single, isolated In atom introduces a strongly localized state $(\mathrm{CBE}+1)$ above the CBE. Further analysis which we present later of the calculated participation ratio for each state confirms this behavior. It can also be seen from Fig. 1(a) that this localized state hybridizes with the CBE: the wave function (WF) for the lowest $\mathrm{CB}$ state is a linear combination of the AlN CBE state and of the localized state $(\mathrm{CBE}+1)$.

Since LDA calculations underestimate the band gap, $\stackrel{23}{\underline{23}}$ we have performed additional DFT calculations within the Heyd-Scuseria-Ernzerhof (HSE) screened exchange hybrid functional scheme, $\stackrel{24}{=}$ implemented in VASP. The same settings as for the LDA calculations have been employed. For HSE-DFT an exact exchange mixing ratio of $\alpha=0.35$ has been used. While this setting slightly

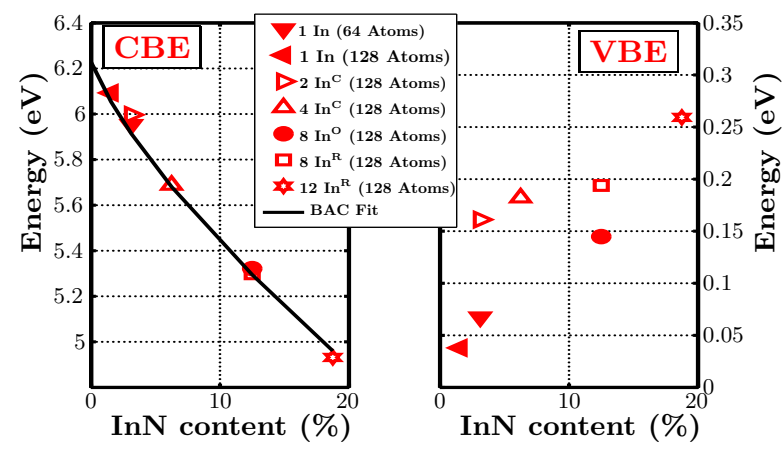

FIG. 2. (Color online) CBE and VBE as a function of In content $x$ in $\mathrm{Al}_{1-x} \operatorname{In}_{x} \mathrm{~N}$ supercells. Calculations for isolated In atoms $\left(\operatorname{In}^{\mathrm{O}}\right)$, and for random $\left(\operatorname{In}^{\mathrm{R}}\right)$ and clustered $\left(\operatorname{In}^{\mathrm{C}}\right)$ configurations. Data is based on HSE-DFT calculations.

overestimates the band gap of InN, $\alpha=0.35$ allows us to describe the energy gap of AlN accurately $\underline{\underline{7}}$ Since we are interested in $\mathrm{Al}_{1-x} \operatorname{In}_{x} \mathrm{~N}$ with low In content $(x<0.2)$, this $\alpha$ value should be appropriate. Again the system was relaxed using the VFF model to address large HSE SCs (64 to 128 atoms). For the lattice parameters we use the results of the HSE-DFT relaxation $\stackrel{25}{\rightleftharpoons}$ which have been modified according to the ratios (relaxed AlN SC vs. relaxed AlInN SC) obtained from the VFF SC calculations. Here, we treat free-standing structures, with no strain field arising from an underlying substrate.

The charge densities from HSE-DFT at $\mathbf{k}=\mathbf{0}$ for CBE and $\mathrm{CBE}+1$ in a 128 atom $\mathrm{SC}$ are shown in Fig. 11 The LDA- (a) and HSE-DFT (c) results show very similar behavior for the CBE state, both giving a WF which has delocalized $\mathrm{CBE}$ character, mixed with a component which is clearly localized around the In atom. We have shown previously for dilute nitride alloys that this mixing of a localized component into the CBE state is a clear signature for the existence of a localized resonant state above the $\mathrm{CBE} \underline{\underline{26}}$ However, because the localized state is resonant with the $\mathrm{CB}$, it can be mixed into several different higher conduction states $\underset{27}{27}$ Because of this mixing, it is therefore not possible in the 128-atom SC calculation to associate a single state above the $\mathrm{CBE}$ with the In resonant state: the charge density of $\mathrm{CBE}+1$ from the HSE-DFT calculation gives a state which is clearly delocalized. The impact of SC size on localization effects has been discussed in detail for example in Ref. 20; it is common to find that a resonant localized state cannot be clearly identified for all SC sizes. However, since HSE- and LDA-DFT give similar results for CBE, LDADFT should also give reliable results for higher CB states. We conclude that the CBE state is in both cases formed through mixing of a resonant In defect state with the host matrix CBE state.

Having discussed the effect of an isolated In atom on the $\mathrm{CB}$, we now present HSE-DFT calculations to analyze the evolution of the band structure with increasing In composition $x$, including the effect of In pairs. Figure 2 shows the calculated $\mathrm{CBE}$ and VBE energies as a 


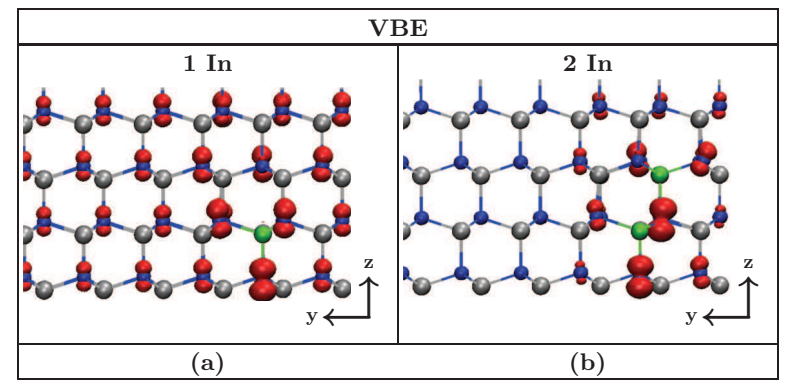

FIG. 3. (Color online) LDA-DFT (1600-atom SC) results for (a) an isolated In atom and (b) an In pair. Dark (red) isosurfaces correspond to $10 \%$ of maximum charge density value.

function of $x$ in $\mathrm{Al}_{1-x} \operatorname{In}_{x} \mathrm{~N}$. We consider structures which include only isolated In atoms (full symbols; referred to as ordered structures) as well as structures which include In pairs and clusters (open symbols). Two types of systems with pairs or clusters are considered: (i) 128-atom SCs with only two or four In atoms, where the In atoms share the same N-atom $\left[2 \mathrm{In}^{\mathrm{C}}\right.$ (128 Atoms), $4 \mathrm{In}^{\mathrm{C}}$ (128 Atoms)], referred to as clustered structures, and (ii) 128atom SCs where eight and 12 In atoms are distributed randomly in the $\mathrm{SC}\left[8 \mathrm{In}^{\mathrm{R}}\right.$ (128 Atoms), $12 \mathrm{In}^{\mathrm{R}}(128$ Atoms)]. For an analysis of higher In concentrations, we refer to our recent semi-empirical tight-binding analysis, $\underline{\underline{4}}$ which is consistent with the here presented DFT data.

The calculated variation of the CBE with $x$ can be well described by a single smooth curve, with $<50 \mathrm{meV}$ difference between any two calculations at the same composition. Turning to the VB, we find that the VBE energies are consistently higher for structures with In pairs or clusters (open symbols) compared to the structures with isolated In atoms only: the VBE shifts upwards by almost $100 \mathrm{meV}$ at $x=3.125 \%$ between the 64 -atom SC with isolated In atoms and the 128-atom SC with an In pair. It is clear that pairs significantly impact the electronic structure of AlInN.

To further analyze the effect of an In pair on the valence band structure, Fig. 3 shows the LDA-DFT VBE charge density calculated in a 1600-atom SC with one In atom (a) and an In atom pair (b). In AlN, due to the negative crystal field splitting and neglecting the weak spin-orbit coupling, the VBE at $\mathbf{k}=\mathbf{0}$ belongs to the irreducible representation $\Gamma_{1} \underline{28}$ The corresponding VBE Bloch state is $p_{z}$-like ${ }^{28}$ The calculated VBE charge density in Fig. 3 reflects this $p_{z}$-like character. We find that even in the one In case, the WF shows signs of localization on the $\mathrm{N}$ sites surrounding the In atom. This localization effect is enhanced strongly in the two In atom case [cf. Fig. 3(b)]. HSE-DFT calculations for a 128-atom SC confirm this result (not shown).

To measure the localization effects more quantitatively, we have calculated the participation ratio (PR), $\mathrm{PR}=V \int|\psi(\mathbf{r})|^{4} \mathrm{~d}^{3} r \stackrel{17}{=}$ Here, $V$ is the volume over which the WF $\psi$ is normalized. The larger the value of $\mathrm{PR}$ for a given volume $V$, the stronger the localization (for a highly localized state, $\mathrm{PR}$ also increases with $V$ ). Within LDA- and HSE-DFT we have calculated PR for the CBE, $\mathrm{CBE}+1$ and VBE states for an isolated In atom and a pair of In atoms sharing a $\mathrm{N}$ neighbor. Table 1 summarizes the normalized $\overline{\mathrm{PR}}=\mathrm{PR} / \mathrm{PR}^{\mathrm{AlN}}$ values, where $\mathrm{PR}^{\mathrm{AlN}}$ is the $\mathrm{PR}$ of the equivalent pure AlN state. We see from Table \that, as soon as one In atom is introduced in AlN, both HSE- and LDA-DFT results show an increase in $\overline{\mathrm{PR}}$ for $\mathrm{CBE}, \mathrm{CBE}+1$ and VBE. Furthermore, from our 1600-atom SC LDA calculation we find that for CBE+1 $\mathrm{PR}$ is almost a factor of 30 larger than $\mathrm{PR}^{\mathrm{AlN}}$. This confirms the formation of a strongly localized In-related state above the CBE, as shown in Fig. 1. Likewise, the introduction of an In pair leads to a strong localization of both the VBE and the CBE WF, as revealed by the $\overline{\mathrm{PR}}$ values in Table $\llbracket$.

To shed further light on these localization effects we focus firstly on the VBE. The localization of the highest valence state due to an In pair is related to the orbital character of the highest valence states (see Fig. 3). As discussed above, the VBE state in AlN is $p_{z}$-like, localized predominantly on the $\mathrm{N}$ atoms. When an In pair is formed, as in Fig. 3(b), the $p_{z}$ orbital on the shared N atom interacts with two neighboring In atoms, leading to a reduced overall bonding interaction for the given $p_{z}$ orbital compared to that for a $p_{z}$ orbital on a $\mathrm{N}$ atom with four $\mathrm{Al}$ neighbors. In addition, the interaction between individual $p_{z}$ orbitals is stronger along the $z$ direction than it is in the $x-y$ plane. This strong directional dependence (in-plane versus out-of-plane) of the interactions between $p_{z}$ orbitals reduces the effective dimensionality of the highest valence states. This anisotropy, coupled with the reduced bonding interaction around In sites, supports the formation of localized states. The impact of the anisotropic interaction can be seen in Fig. 3(b), where there is a rapid reduction of the VBE probability density in the $x-y$ plane in the presence of an In pair, with a slower decay of the VBE probability density along the $z$ direction, due to the stronger interaction of $p_{z}$ orbitals along that axis.

Recently, valence state localization due to In-N-In chains has been observed experimentally by Chichibu et $a l$. in GaInN structures $\stackrel{16}{\underline{16}}$ First-principles calculations carried out for zinc blende GaInN on 8-atom $\frac{17}{}$ and 64 atom SCs with a few selected In configurations $\frac{18}{18}$ show hole localization when the In impurities are clustered to form a zig-zag In-N-In-N-In chain $\underline{18}$. Liu et al ${ }^{19} \underline{\underline{19}}$ studied wurtzite (WZ) InGaN systems based on DFT calculations. Special attention was paid to the impact of uniformly distributed In, an In pair and a condensate where In atoms congregate together. The authors conclude that short In-N-In chains lead to a weak localization of va-

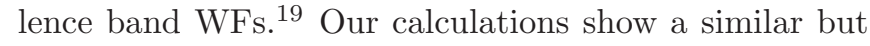
even stronger effect in AlInN, with localization observed even when just a single In pair is introduced into a large AlN SC. Therefore, we suggest an experiment similar to that performed by Chichibu et al $\stackrel{16}{n}$ for GaInN, to study 
TABLE I. Normalized CBE, CBE +1 and VBE WF participation ratio $\overline{\mathrm{PR}}$ for one In atom and an In atom pair in AlN. LDA-DFT calculations have been performed on a 1600-atom SC; HSE-DFT are obtained from a SC with 128 atoms.

\begin{tabular}{cccccc}
\hline \multirow{2}{*}{ PR } & \multicolumn{3}{c}{ Isolated In atom } & \multicolumn{2}{c}{ Pair of In atoms } \\
\cline { 2 - 6 } & CBE & CBE+1 & VBE & VBE & CBE \\
\hline HSE-DFT & 1.26 & 1.15 & 1.23 & 1.57 & 1.63 \\
LDA-DFT & 1.18 & 27.56 & 1.05 & 2.51 & 2.75 \\
\hline
\end{tabular}

the localization effects in AlInN.

The variation of the CBE energy with composition $x$ in III-V alloys where one of the alloy components introduces resonant defect states above the $\mathrm{CBE}$ can be described by a band-anticrossing (BAC) model 26,29,30 Here, the evolution of the CBE energy is given by the lower eigenvalue of the $2 \times 2$ Hamiltonian matrix 29,30

$$
H(x)=\left(\begin{array}{cc}
E_{\mathrm{D}}(x) & V_{\mathrm{D}, \mathrm{c}} \\
V_{\mathrm{D}, \mathrm{c}} & E_{\mathrm{c}}(x)
\end{array}\right),
$$

where $E_{\mathrm{D}}$ is the resonant defect state energy, which corresponds to $\mathrm{CBE}+1$ in Fig. 1(b). $E_{\mathrm{c}}(x)$ describes the virtual crystal approximation (VCA) variation of the host matrix CBE state with composition $x$, and $V_{\mathrm{D}, \mathrm{c}}$ describes the interaction between the levels, which varies with $x$ as $\beta \sqrt{x}$, where the $\sqrt{x}$ dependence arises because the interaction is between localized defect and extended conduction states 31 Thus, the energies of the CBE $\left(E_{-}\right)$and the higher lying resonant state $\left(E_{+}\right)$change according to $E_{ \pm}=(1 / 2)\left(E_{\mathrm{c}}+E_{\mathrm{D}}\right) \pm \sqrt{(1 / 4)\left(E_{\mathrm{c}}-E_{\mathrm{D}}\right)^{2}+V_{\mathrm{D}, \mathrm{c}}^{2}}$. Our 1600-atom LDA-DFT calculations on structures containing a $\mathrm{N}$ atom with 2,3 or 4 In neighbors show that such pairs and clusters also give rise to a resonant defect state, which shifts down in energy but is calculated to still remain above the CBE with increasing cluster size. We therefore take $E_{\mathrm{D}}$ to vary as $E_{\mathrm{In}}^{0}-\alpha x$, to reflect the increasing number of In pairs and clusters expected as $x$ increases. Likewise the CBE varies in the $\mathrm{VCA}$ as $E_{\mathrm{c}}^{0}-\gamma x$. The solid black line in Fig. 2 shows a BAC fit to the variation of the CBE with composition $x$, calculated with the In resonant state placed $0.7 \mathrm{eV}$ above the AlN CBE (estimated from the LDA data), and as- suming $\beta=2.5 \mathrm{eV}$, and $\alpha=\gamma=2.3 \mathrm{eV}$. Clearly, with four free parameters, there is a wide range of other values that could have been chosen. The values used here are comparable with those previously used for $\mathrm{GaN}_{x} \mathrm{As}_{1-x}, 26$ supporting that the evolution of the CBE in $\mathrm{Al}_{1-x} \operatorname{In}_{x} \mathrm{~N}$ is well described using a BAC model to take account of the interaction between the localized In states and the host matrix CBE.

Our finding of a BAC interaction in AlInN is further supported by recent experimental data and DFT calculations showing a marked reduction in the band gap deformation potential in AlInN for low In compositions. ${ }^{32}$ Localized states such as the In isoelectronic defect level in AlN typically have a smaller hydrostatic deformation potential than the CBE. Because the lowest CB state is formed by mixing such localized state character with the host matrix CBE, this then leads to a measurable reduction in the direct gap deformation potential. 29

In conclusion, based on DFT studies we have demonstrated that cation-induced localized states strongly perturb the band structure of the III-V alloy $\mathrm{Al}_{1-x} \operatorname{In}_{x} \mathrm{~N}$, leading to the breakdown of the assumption of a single composition-independent band gap bowing parameter. We have shown that In-related localized states are formed both in the $\mathrm{CB}$ and the $\mathrm{VB}$ in $\mathrm{Al}_{1-x} \operatorname{In}_{x} \mathrm{~N}$ for low In composition, and that these localized states dominate the evolution of the band structure with increasing $x$.

This work was supported by Science Foundation Ireland (project No. 10/IN.1/I2994) and the EU 7th Framework Programme (ALIGHT; FP7-280587). We thank Peter Parbrook and Emanuele Pelucchi for very useful discussions.
* stefan.schulz@tyndall.ie

1 S. Pandey, D. Cavalcoli, B. Fraboni, A. Cavallini, T. Brazzini, and F. Calle, Appl. Phys. Lett. 100, 152116 (2012).

2 J. Wu, J. Appl. Phys. 106, 011101 (2009).

3 I. Vurgaftman, J. R. Meyer, and L. R. Ram-Mohan, J. Appl. Phys. 89, 5815 (2001).

${ }^{4}$ K. Wang, R. W. Martin, D. Amabile, P. R. Edwards, S. Hernandez, E. Nogales, K. P. O'Donnell, K. Lorenz, E. Alves, V. Matias, A. Vantomme, D. Wolverson, and I. M. Watson, J. Appl. Phys. 103, 073510 (2008); E. Sakalauskas, H. Behmenburg, C. Hums, P. Schley, G. Rossbach, C. Giesen, M. Heuken, H. Kalisch, R. H. Jansen, J. Bläsing, A. Dadgar, A. Krost, and R. Goldhahn, J. Phys. D: Appl. Phys. 43, 365102 (2010); S. Schulz,
M. A. Caro, L.-T. Tan, P. J. Parbrook, R. W. Martin, and E. P. O'Reilly, Appl. Phys. Express 6, 121001 (2013).

5 I. Vurgaftman and J. R. Meyer, J. Appl. Phys. 94, 3675 (2003); R. R. Pel, C. Caetano, M. Marques, L. G. Ferreira, J. Furthmüller, and L. K. Teles, Appl. Phys. Lett. 98, 151907 (2011).

6 M. A. Caro, S. Schulz, and E. P. O'Reilly, Phys. Rev. B 88, 214103 (2013).

7 P. G. Moses, M. Miao, Q. Yan, and C. G. Van de Walle, J. Chem. Phys. 134, 084703 (2011).

8 K. S. Kim, A. Saxler, P. Kung, M. Razeghi, and K. Y. Lim, Appl. Phys. Lett. 71, 800 (1997).

9 T. Aschenbrenner, H. Dartsch, C. Kruse, M. Anastasescu, M. Stoica, M. Gartner, A. Pretorius, A. Rosenauer, 
T. Wagner, and D. Hommel, J. Appl. Phys. 108, 063533 (2010).

${ }^{10}$ W. Walukiewicz, W. Shan, K. M. Yu, J. W. Ager, E. E. Haller, I. Miotkowski, M. J. Seong, H. Alawadhi, and A. K. Ramdas, Phys. Rev. Lett. 85, 1552 (2000).

11 P. D. C. King, T. D. Veal, P. H. Jefferson, C. F. McConville, T. Wang, P. J. Parbrook, H. Lu, and W. J. Schaff, Appl. Phys. Lett. 90, 132105 (2007).

12 J. R. Waldrop and R. W. Grant, Appl. Phys. Lett. 68, 2879 (1996).

13 S.-H. Wei and A. Zunger, Appl. Phys. Lett. 69, 2719 (1996).

14 A. Rizzi, R. Lantier, F. Monti, H. Lüth, F. D. Sala, A. D. Carlo, and P. Lugli, J. Vac. Sci. Technol. B 17, 1674 (1999).

15 P. D. C. King, T. D. Veal, C. E. Kendrick, L. R. Bailey, S. M. Durbin, and C. F. McConville, Phys. Rev. B 78, 033308 (2008).

16 S. F. Chichibu, A. Uedono, T. Onuma, B. A. Haskell, A. Chakraborty, T. Koyama, P. T. Fini, S. Keller, S. P. DenBaars, J. S. Speck, U. K. Mishra, S. Nakamura, S. Yamaguchi, S. Kamiyama, H. Amano, I. Akasaki, J. Han, and T. Sota, Nature Mater. 5, 810 (2006).

17 B. Lee and L. W. Wang, J. Appl. Phys. 100, 093717 (2006).

18 X. Wu, E. J. Walter, A. M. Rappe, R. Car, and A. Selloni, Phys. Rev. B 80, 115201 (2009).

19 Q. Liu, J. Lu, Z. Gao, L. Lai, R. Qin, H. Li, J. Zhou, and G. Li, Phys. Stat. Sol B 247, 109 (2010).

20 J. A. Chan, J. Z. Liu, and A. Zunger, Phys. Rev. B 82, 045112 (2010).
21 G. Kresse and J. Furthmüller, Phys. Rev. B 54, 11169 (1996).

22 R. W. Martin, Phys. Rev. B 6, 4546 (1972).

23 J. P. Perdew and M. Levy, Phys. Rev. Lett. 51, 1884 (1983).

24 J. Heyd, G. E. Scuseria, and M. Ernzerhof, J. Chem. Phys. 118, 8207 (2003); J. Heyd and G. E. Scuseria, ibid. 121, 1187 (2004).

25 M. A. Caro, S. Schulz, and E. P. O'Reilly, Phys. Rev. B 86, 014117 (2012).

26 E. P. O'Reilly, A. Lindsay, P. J. Klar, A. Polimeni, and M. Capizzi, Semicond. Sci. Technol. 24, 033001 (2009).

27 A. Lindsay and E. P. O'Reilly, Solid State Comm. 118, 313 (2001).

28 G. F. Koster, J. O. Dimmock, R. G. Wheeler, and H. Statz, Properties of thirty-two point groups (M.I.T. Press, Massachusetts, 1969).

29 W. Shan, W. Walukiewicz, J. W. Ager, E. E. Haller, J. F. Geisz, D. J. Friedman, J. N. Olson, and S. R. Kurtz, J. Appl.Phys. 86, 2349 (1999).

30 A. Lindsay and E. P. O'Reilly, Phys. Rev. Lett. 93, 196402 (2004).

31 A. Lindsay and E. P. O'Reilly, Solid State Commun. 112, 443 (1999).

32 I. Gorczyca, A. Kaminska, G. Staszczak, R. Czernecki, S. P. Lepkowski, T. Suski, H. P. D. Schenk, M. Glauser, R. Butte, J. F. Carlin, E. Feltin, N. Grandjean, N. E. Christensen, and A. Svane, Phys. Rev. B 81, 235206 (2010). 